\title{
La tecnología en el ámbito educativo ante el COVID: una apuesta por los MOOC como estrategia formativa en el contexto universitario
}

Technology in the educational field before COVID: a commitment to MOOCs as a training strategy in the university context

\section{María del Mar Fernández-Martínez. Universidad de Almería. mfm386@ual.es}

Antonio Hilario Martín-Padilla. Universidad Pablo de Olavide. ahmarpad@upo.es

Antonio Luque de la Rosa. Universidad de Almería. aluque@ual.es

Isabel Ana Eguizábal-Román. Miembro del Grupo de Investigación Edulnnovagogía@ (HUM-971). iselaeguizabal@gmail.com

\section{RESUMEN.}

Si bien el desarrollo de la tecnología en el ámbito educativo ha estado promoviendo cambios progresivos en la metodología didáctica, sobre todo a nivel universitario, en el último cuarto de siglo, es a raíz de la pandemia que azota a la humanidad (COVID-19) cuando desde el contexto académico se ha visto la necesidad imperiosa de repensar todo el funcionamiento y posibilidades no presenciales basadas en internet a través de docencia síncrona o asíncrona. Así, con el desarrollo de los MOOC se han visto grandes posibilidades de atender las demandas educativas de manera no presencial, posibilitando la atención individualizada y abierta al conjunto de alumnado que presenten casuísticas y necesidades variadas.

En el presente trabajo, pretendemos llevar a cabo un acercamiento a los MOCC en cuanto conceptualización, características, elementos y tipologías, profundizando en su relación con la atención educativa en el momento pandémico que nos circunda.

Para terminar, realizaremos un recorrido por diversos agregadores/buscadores de MOCC y ejemplificaremos varios de ellos para demostrar su potencialidad educativa.

\section{PALABRAS CLAVE.}

Tecnología, Educación, MOOC, COVID-19, Teleformación.

\section{ABSTRACT.}

Although the development of technology in the educational field has been promoting progressive changes in teaching methodology, especially at the university level, in the last quarter century, it is due to the pandemic that plagues humanity (COVID-19) when from the

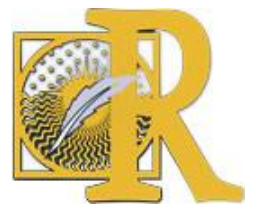


academic context there has been the urgent need to rethink all the operation and non-contact possibilities based on the Internet through synchronous or asynchronous teaching.

Thus, with the development of MOOCs, great possibilities have been seen to meet educational demands in a non-face-to-face way, enabling individualized and open attention to the group of students who present cases and varied needs.

In the present work, we intend to carry out an approach to MOCCs in terms of conceptualization, characteristics, elements and typologies, deepening their relationship with educational care in the pandemic moment that surrounds us.

To finish, we will make a tour of various MOCC aggregators / search engines and we will copy several of them to demonstrate their educational potential.

\section{KEY WORDS.}

Technology, Education, MOOC, COVID-19, Teleformation.

\section{Los retos de la educación ante el COVID-19.}

La actual pandemia que azota a la comunidad internacional ha generado una lógica preocupación por parte de todas las instituciones. Esta preocupación cuenta con una doble vertiente contrapuesta. Por una parte, existe una clara dificultad de hacer frente al imperativo de aislamiento y confinamiento para salvaguardar el estado de salud de la población, en contraposición se presenta la necesidad estructural de mantener el desarrollo económico y social de las regiones afectadas (Bao, Sun, Meng, Shi \& Lu, 2020; Ruiz-Cruz, 2020).

En este sentido son numerosos los nuevos frentes que demandan de atención, ideas y soluciones que sean capaces de combatir los efectos de la falta de relación social presencial en diversos planos.

Desde inicios de marzo el cierre de centros educativos fue aumentando de forma paulatina en los diferentes países afectados por esta crisis sanitaria. En todo ese tiempo, más de 60 millones de docentes no pueden acudir a sus aulas, dejando así de poder atender de forma normalizada a más de mil millones de alumnas y alumnos de escuelas, institutos y universidades en todo el mundo (ONU, 2020, UNESCO, 2020a; UNESCO, 2020b).

No debemos olvidar que la educación es un derecho inalienable, que debe ser objeto de protección, especialmente cuando las circunstancias son más lesivas para la población. Y, precisamente, frente a las repercusiones psicosociales que subyacen a las situaciones de crisis, la Educación debe actuar como agente protector, ofreciendo esperanza a la población y convirtiéndose en un elemento estructural estabilizador con una clara proyección hacia el desarrollo local futuro (UNICEF, 2020).

Así, ante la problemática económica y psicológica surgida a nivel general a raíz del aislamiento (aumento del paro, depresión, estrés, etc.), desde la comunidad educativa han surgido múltiples iniciativas desde las que se han planteado estrategias con las que poder continuar, de la manera más normalizada posible, el proceso educativo del alumnado (Brooks et al. 2020). En este contexto, la comunidad educativa ha realizado un ingente esfuerzo por tecnologizarse, por avanzar hacia la Sociedad de la información y el Conocimiento, si bien a una velocidad mucho menor de la que la situación de crisis demandaba.

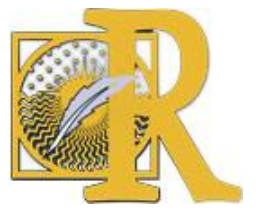


Esfuerzos e iniciativas como estas son cada vez más necesarias, máxime cuando, en vísperas casi de comenzar un nuevo curso académico tras el periodo estival, están apareciendo rebrotes de COVID-19 en diferentes países, lo cual genera enormes dudas, que crecen a diario, con respecto a la forma en que se podrá afrontar el nuevo curso y el nivel de presencialidad que podrá adoptarse.

En este sentido, en los últimos tiempos, ha habido una apuesta importante por actividades, cursos y estrategias formativas que basan su metodología y el uso de recursos en un desarrollo tecnológico que favorece la no presencialidad y permite la atención a grupo muy numeroso de alumnado de forma simultánea (Castaño-Muñoz, Duart \& Teresa, 2015; Colorado, Marín-Díaz \& Zavala, 2016; Estévez \& García 2015; Roig-Vila, Mondéjar \& Lorenzo-Lledó, 2016). En este escenario es donde surgen y se desarrollan cada vez más los denominados MOOC.

\section{Los MOOC: nuevos senderos para la innovación educativa.}

El acrónimo «MOOC» significa, según sus siglas en inglés, Massive Open Online Course (Rheingold, 2013), y se refiere a una tipología de cursos que son Masivos en Línea y Abiertos. Lógicamente, sus características más destacables serían precisamente:

- Carácter abierto, con la posibilidad de que, a priori, pueden realizarlos cualquier persona, ya que no hay requisitos académicos.

- Online, de manera que toda la información y relación entre actores se lleva a cabo a través de Internet.

- Masivo, en el sentido de que pueden ser miles el número de personas que asisten a estos cursos.

La metodología didáctica de los MOOC supone un revulsivo a nivel pedagógico al avanzar en la corresponsabilidad del aprendizaje entre docente y alumnado, convirtiéndose este último en generador de contenido y de conexiones entre los diversos elementos del curso.

Al mismo tiempo, se intenta potenciar en los MOOC, la relación y uso de las diferentes redes sociales (Facebook, Twitter, etc.), para fomentar la interactividad y aprovechar el uso de estas para agregar contenidos, compartir información o estrategias de aprendizaje (MéndezGarcía, 2015).

En por esto que autores como Vázquez-Cano y López Meneses (2015), señalan que los nuevos escenarios formativos universitarios están siendo orientados hacia un nuevo modelo de formación masiva, abierta y gratuita por medio de una metodología basada en la videosimulación y el trabajo colaborativo del estudiantado.

Así, los MOOC se están convirtiendo en una nueva modalidad formativa basada en ecologías digitales, respondiendo al hecho de que las Tecnologías de la Información y la comunicación están revolucionando los procesos formativos y comunicativos con sus avances y evoluciones (Vázquez-Cano et al., 2020).

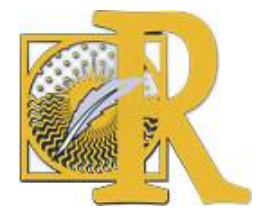


Figura 1: Características de los MOOC

\begin{tabular}{|c|c|c|c|}
\hline \multicolumn{4}{|c|}{ Características de los cursos MOOC } \\
\hline $\begin{array}{l}\text { Es masivo no como resultado } \\
\text { (como lo es un canal de TV, una } \\
\text { emisora de radio, etc., que llegan } \\
\text { a una gran cantidad de gente), } \\
\text { sino porque tiene interactividad } \\
\text { entre muchas personas. }\end{array}$ & $\begin{array}{l}\text { Es abierto: lo } \\
\text { que implica } \\
\text { gratuidad y } \\
\text { que sea libre, } \\
\text { que se pueda } \\
\text { ir y venir. }\end{array}$ & $\begin{array}{l}\text { Es on line: porque se } \\
\text { crea conexión entre } \\
\text { diferentes personas } \\
\text { del mundo a través de } \\
\text { la Red. }\end{array}$ & $\begin{array}{l}\text { Es un curso: no una } \\
\text { comunidad, ni un evento, ni } \\
\text { un ambiente, sino que, como } \\
\text { los cursos, comienza, } \\
\text { transcurre y termina. }\end{array}$ \\
\hline
\end{tabular}

Fuente: Adaptado de "Actas del VII Encuentro Internacional de Educación de Fundación Telefónica", de Downes, 2013, Caracas, Venezuela.

En cuanto a sus características, además de las ya mencionadas, hay que señalar que un MOOC suele venir configurado por los siguientes elementos:

- Wiki, donde se planifica el curso (por semanas, temas, etc.).

- Lista de correo para suscribirse.

- Blog del curso, en el cual los participantes opinan y participan cada día sobre el aprendizaje realizado.

- Ambiente online, en el que se lleva a cabo la interacción entre el alumnado.

En la situación actual de pandemia, al no poder garantizarse el acceso, formación y desarrollo en el conjunto de la ciudadanía, puede convertirse en un factor que incremente desproporcionalmente el grado de exclusión social y educativa ya existente de determinadas zonas (Cabero-Almenara \& Ruiz-Palmero, 2018). Y lo que puede ser aún más preocupante, pueden verse afectadas zonas donde no existían índices relevantes de exclusión social y educativa. En este contexto, los MOOC pueden favorecer un desarrollo tecnológico, didáctico y de inclusión social y educativa gracias a su carácter de cursos de carácter masivos, abiertos y en línea.

Al hablar de MOOC debemos también señalar que existen diferentes tipos. Aunque existe una amplia tipología, en términos generales, suelen clasificarse en dos tipos: XMOOC y cMOOC (Department for Business, Innovation and Skills, 2013; Downes, 2012; GómezGalán, Martín-Padilla, Bernal-Bravo \& López-Meneses, 2017; Hill, 2012; Siemens, 2012; Vázquez-Cano, López Meneses y Sarasola, 2013).

Figura 2. Diferencias entre cMOOC y xMOOC.

\begin{tabular}{|l|l|}
\hline \multicolumn{1}{|c|}{$\mathrm{CMOOC}$} & \multicolumn{1}{c|}{$\mathrm{xMOOC}$} \\
\hline Aprendizaje en red & Enfoque tradicional \\
\hline No lineal, caótico & Lineal \\
\hline Aprendizaje individual & Aprendizaje conceptual \\
\hline Conocimiento distribuido & Contenidos comunes \\
\hline Red escalable & No escalable \\
\hline Aprendizaje activo & Aprendizaje pasivo \\
\hline
\end{tabular}


En otra clasificación, realizada por Downes (2012), se establecen los siguientes tipos:

a. Los MOOC basados en contenidos, con poca interactividad.

b. Los MOOC basados en redes, donde no hay una ruta predefinida y única. Así, aunque sea masivo es personal ya que cada persona elige aquello que se adapte más a su experiencia y situación de partida.

c. Los MOOC híbridos: incluyen una red para facilitar la diversificación, pero las tareas están basadas en un contenido.

A continuación, en las siguientes líneas, se realizará una breve descripción de diversos MOOC orientados al conocimiento y la investigación sobre la pandemia originada por el COVID-19. Para ello hemos seleccionados las principales plataformas agregadoras de cursos MOOC (SCOPEO, 2013; Vázquez-Cano et al., 2013; Martín-Padilla, Bernal-Bravo, RamírezFernández y López-Meneses, 2016) ${ }^{1}$.

Cabe decir que en la fecha de realización del presente artículo (mayo-junio de 2020) el número de cursos MOOC con el keyword: COVID-19, coronavirus, virus y pandemia fueron: 120 cursos (Plataforma Coursera); 7 cursos (Plataforma EdX) y 2 cursos (Plataforma Miriadax).

Dada la extensión limitada de este artículo se incluirán solo aquellos MOOC más representativos, con objeto de ejemplificar su funcionamiento e incardinación en el mundo académico de la Enseñanza Superior.

\section{COVID-19: COMUNICARNOS SIN DAÑO DURANTE LA PANDEMIA. Estructura del curso.}

El comunicador y todos los medios de comunicación se constituyen en agentes fundamentales en la prevención del escalamiento de la epidemia actual y en proveer información que no cause daño y que beneficie a todos.

- Módulo 1. Generalidades sobre comunicarnos sin daño.

- Módulo 2. El comunicador como vector de salud.

\section{Institución que lo imparte.}

Universidad Pontificia Javeriana (Colombia).

Enlace: https://www.edx.org/course/comunicacion-sin-dano-durante-la-pandemia

\section{Breve descripción.}

Es muy importante tener en cuenta que la manera en que nos comunicamos puede afectar la salud mental de las personas. La comunicación es muy importante en todo momento, pero especialmente en estos momentos de pandemia, el saber cómo comunicarnos ahora puede generar salud, malestar, trastornos o problemas.

Por este motivo y conscientes de la responsabilidad social de la academia y comprometidos con las necesidades educativas del momento. Las Facultades de Medicina y Comunicación y Lenguaje de la Pontificia Universidad Javeriana, desarrollaron el curso virtual Comunicarnos sin daño durante la pandemia, en el cual se tratarán las generalidades sobre comunicarnos sin daño, cómo afecta la comunicación los estamentos psíquicos de la persona y cómo el comunicador puede ayudar a dar salud mental.

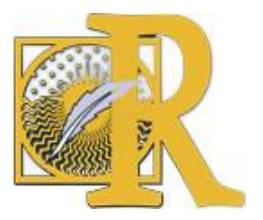




\section{HABILIDADES DE RESILIENCIA.}

\section{Estructura del curso.}

- Módulo 1: Resiliencia y optimismo.

- Módulo 2: Enfoques cognitivos de la resiliencia: estrategias para aumentar el optimismo y el pensamiento resiliente.

- Módulo 3: Manejando la ansiedad y aumentando las emociones positivas como la gratitud.

- Módulo 4: Aprovechando las fortalezas del carácter y fortaleciendo las relaciones.

\section{Institución que lo imparte.}

\section{Universidad de Pensilvania.}

Enlace: https://es.coursera.org/learn/resilience-uncertainty\#about

\section{Breve descripción.}

Todos enfrentamos desafíos diferentes y difíciles a medida que enfrentamos la pandemia de COVID-19. Para apoyarlo en este momento de incertidumbre, la Universidad de Pensilvania está compartiendo esta versión gratuita y única del curso de la Dra. Karen Reivich de los Fundamentos de Especialización de Psicología Positiva.

Aprenda a incorporar intervenciones de resiliencia en su vida personal y profesional con la Dra. Karen Reivich. En este curso, está expuesto a la investigación fundamental en resiliencia, que incluye factores protectores como la agilidad mental y el optimismo. Se exploran varios tipos de intervenciones de resiliencia, incluidas las estrategias cognitivas; estrategias para manejar la ansiedad y aumentar las emociones positivas como la gratitud; $y$ una habilidad crítica para mejorar las relaciones. A lo largo del curso, escuchará ejemplos de personas que usan habilidades de resiliencia en sus vidas personales y profesionales.

\section{INGLÉS BÁSICO: CONVERSACIONAL Y NETWORKING.}

\section{Estructura del curso.}

Cumple tu meta de aprender inglés básico para utilizarlo de forma estratégica en los negocios e incrementar tu éxito profesional.

Módulo 1. ¡Mucho gusto!

- Presentarte y dar información personal.

- Hacer preguntas sobre cosas y personas.

- Sugerir cosas para hacer.

- Preguntar sobre objetos.

- Hablando de posesiones.

- Encontrar cosas en común y diferencias.

- Gustos y disgustos.

Módulo 2. Rutinas.

- Hablar sobre hábitos.

- Comprando cosas.

- Preguntar sobre información de viaje.

- Vocabulario de viaje y preguntas. 
Módulo 3. Viviendo el pasado.

- Hablando de situaciones.

- Averiguando dónde estaban las personas en el pasado.

- Hacer y responder preguntas sobre el pasado.

- Dando opiniones.

Módulo 4. Mis planes futuros.

- Hablando de planes.

- Ir a nuevos lugares, hacer cosas nuevas y enfrentar oportunidades de negocios.

- Iniciar y finalizar una conversación.

\section{Institución que lo imparte.}

Red de Universidades Anáhuac.

Enlace:

https://www.edx.org/course/fundamentos-de-ingles-basico-conversacional-y-

networking

\section{Breve descripción.}

En tu vida diaria y laboral, probablemente te has enfrentado a situaciones en donde tienes que leer, entender o hablar inglés y te encuentras en una encrucijada cuando no te sientes capaz de realizarlo de manera efectiva.

$\mathrm{Si}$ te sentiste identificado, este contenido fue creado para ti. Con este curso en línea podrás aprender inglés de forma eficaz para desenvolverte correctamente en tu vida diaria y laboral, eliminando la barrera del idioma para alcanzar el éxito profesional.

Con más de 30 años de experiencia, los docentes han desarrollado este curso especialmente para que puedan enfrentar las situaciones de la vida cotidiana, a través de una metodología mixta; aplicando el idioma mediante ejercicios que alientan la conexión entre los significados, situaciones y repetición, facilitando tu comunicación en este idioma.

\section{REIMAGINAR EL TRABAJO: ESTRATEGIAS DURANTE COVID-19 Y MÁS ALLÁ. Estructura del curso:}

1. Aprenda y evalúe cómo trabajar con otros a distancia.

2. Aprenda y aplique estrategias de diseño centradas en el ser humano para resolver desafíos de colaboración de trabajo remoto.

3. Ejercer el pensamiento visual para crear prototipos, probar y recibir comentarios.

\section{Institución que lo imparte.}

The University of British Columbia.

Enlace: https://www.edx.org/course/working-remotely-and-covid-19

\section{Breve descripción.}

¡Mejore y reinvente el trabajo a distancia durante COVID-19 y más allá! Aprenda de expertos en diseño estratégico y cree su kit de herramientas en el hogar mediante la aplicación de técnicas de diseño centradas en el ser humano.

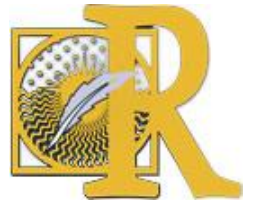


Como resultado de COVID-19, millones de nosotros nos estamos adaptando para trabajar desde casa, puede que hayamos perdido nuestros trabajos o estamos buscando formas de reinventar nuestro trabajo para cumplir con los requisitos de distanciamiento social.

- Diseñado para individuos y personas que administran organizaciones pequeñas y medianas, este curso introductorio lo inspirará a reimaginar cómo puede transformar su trabajo con otros desde la distancia, utilizando herramientas de diseño UX centradas en el ser humano que se han aplicado en varios diseños orientados empresas por décadas.

- Lleno de conversación experta, diferentes herramientas y mapas visuales reflexivos que puede aplicar a su propio contexto, este curso básico es útil para cualquiera que intente reinventar su trabajo desde casa.

- Si disfruta del curso y desea desarrollar una comprensión más profunda de este tema, le recomendamos que se inscriba posteriormente en el programa de certificado profesional edX de dos cursos. El certificado se basa en las enseñanzas de este curso introductorio, que ofrece estudios de casos de la vida real, interacción con el instructor en vivo y comentarios de expertos.

\section{PIVOTANDO A LA ENSEÑANZA EN LÍNEA: PERSPECTIVAS DE INVESTIGACIÓN Y PROFESIONALES.}

\section{Estructura del curso.}

Este curso explora prácticas efectivas basadas en la investigación para la enseñanza y el aprendizaje en línea, brindando orientación sobre cómo pivotar los cursos existentes en línea mientras se mejora el éxito y la participación de los estudiantes.

Módulo 0: Preparación para el curso.

Módulo 1: Comenzando rápidamente.

Módulo 2: Creación de contenido.

Módulo 3: Fomento de la interacción.

Módulo 4: Evaluación.

Módulo 5: Avanzando y Aprendizaje combinado.

\section{Institución que lo imparte.}

University of Texas Arlington.

Enlace: $\quad$ https://www.edx.org/es/course/pivoting-to-online-teaching-research-andpractitioner-perspectives?

\section{Breve descripción.}

En este curso, los participantes explorarán prácticas efectivas basadas en investigaciones para la enseñanza y el aprendizaje en línea. Al inscribirse, aprenderá formas prácticas de pasar rápidamente a la enseñanza en línea, guiado por los mejores académicos y profesionales en el campo. En cada módulo, verá videos y leerá artículos de expertos en aprendizaje en línea y participará en actividades y debates que abarcarán temas críticos que harán del entorno en línea una experiencia de aprendizaje enriquecedora para sus estudiantes. Los instructores sintetizarán recursos relevantes para ayudar a aquellos que son nuevos en el aprendizaje en línea y aquellos que tienen experiencia, pero desean expandir

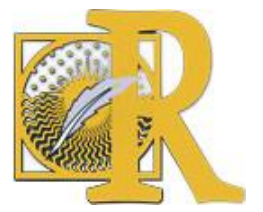


sus habilidades y brindar apoyo a otros. Tendrá la oportunidad de hacer preguntas, compartir prácticas que han funcionado bien en entornos de aprendizaje en línea y recibir comentarios sobre sus planes de enseñanza y aprendizaje.

Dados los recientes desarrollos mundiales relacionados con COVID-19, muchos han cambiado rápidamente para mover la enseñanza en línea. Para aquellos que no han enseñado en línea antes, esta puede ser una experiencia desafiante. Afortunadamente, hay una rica base de investigación, que data de más de sesenta años, que proporciona información y orientación sobre los factores clave que permiten un aprendizaje exitoso en línea. Este curso apoyará el giro hacia el aprendizaje en línea mediante la exploración de la literatura científica, así como las acciones prácticas que permiten el éxito en línea y resultados equitativos para todos los alumnos.

Si bien el público objetivo del curso son las instituciones postsecundarias, este curso será de utilidad para cualquier persona que ingrese a la enseñanza y el aprendizaje en línea.

\section{EL FUTURO DEL TRABAJO: PREPARACIÓN PARA LA INTERRUPCIÓN. \\ Estructura del curso.}

Solo el MOOC explorará el Informe sobre el desarrollo mundial (WDR) de 2019 y la fuerza laboral del futuro. Descubra cómo la IA, la automatización, las tecnologías avanzadas y las nuevas empresas están cambiando el mundo.

Semana 1 - Naturaleza cambiante del trabajo

Semana 2 - Capital humano: un nuevo marco

Semana 3 - Aprendizaje permanente: desde el nacimiento hasta la jubilación

Semana 4 - Regreso al trabajo y protección social

Semana 5 - Invertir en inclusión social

\section{Institución que lo imparte.}

Open Learning Campus (World Bank Group).

Enlace: $\quad$ https://es.tun.com/cursos/El-futuro-del-trabajo-prepar\%C3\%A1ndose-para-lainterrupci\%C3\%B3n/wbgx/

\section{Breve descripción.}

Los trabajadores del futuro necesitarán nuevos conjuntos de habilidades para competir. Los recientes avances en tecnología están cambiando la forma en que vivimos, nos comunicamos y hacemos negocios, interrumpiendo las industrias tradicionales y redefiniendo la relación empleado-empleador.

La automatización, la inteligencia artificial y la hiperconectividad digital eliminarán miles de trabajos rutinarios y de baja calificación. Sin embargo, estos mismos avances presentan nuevas oportunidades, como:

- Nueva creación de empleo.

- Productividad incrementada

- Prestación mejorada de servicios públicos.

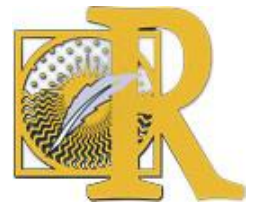


Este curso enfatiza la urgencia de desarrollar capital humano para enfrentar los desafíos de las próximas décadas. Los países en desarrollo deberán tomar medidas rápidas para invertir en su gente a medida que la innovación continúe acelerándose. Los temas en el curso incluyen: inteligencia artificial, economía del trabajo, mundo del trabajo, el futuro del trabajo, mercado laboral, formuladores de políticas, nuevas tecnologías, economía digital, trabajos del futuro, aprendizaje automático y fuerza laboral.

Ejemplo basado en habilidades:

Los participantes aprenderán a través de charlas tipo Ted, podcasts, lecturas, cuestionarios interactivos y ejercicios basados en escenarios. También tendrán la oportunidad de compartir y dialogar con colegas y expertos, incluidos profesionales, funcionarios gubernamentales, académicos y del sector privado. Este curso presentará a los estudiantes enfoques innovadores que desarrollarán las nuevas habilidades requeridas en el siglo XXI, que incluyen:

- Análisis crítico.

- Resolución de problemas.

- "Habilidades blandas" como trabajo en equipo y empatía.

\section{SER MÁS CREATIVOS.}

Estructura del curso.

1. Acerquémonos a la creatividad

2. ¿Qué detiene y qué promueve la creatividad?

3. Estrategias para ser más creativos

4. Heurísticas y modelos

5. Algunos elementos sorprendentes

\section{Institución que lo imparte.}

Universidad Nacional Autónoma de México

Enlace: https://www.edx.org/course/comunicacion-sin-dano-durante-la-pandemia

\section{Breve descripción.}

¡Claro que todos podemos potenciar nuestra creatividad a través de procedimientos, rutinas y protocolos sencillos! El participante desarrollará su talento creativo para aportar soluciones originales y generar ideas y productos en el ámbito en que se desenvuelve y en la vida cotidiana.

\section{PRIMEROS AUXILIOS PSICOLÓGICOS (PAP). EDICIÓN ESPECIAL COVID-19 Estructura del curso}

- Módulo 1. Definición y condiciones de aplicación

- Módulo 2. PAP en niños de hasta 12 años

- Módulo 3. PAP en adolescentes y adultos

- Módulo 4. PAP en colectivos vulnerables

- Módulo 5. Autocuidado del interviniente

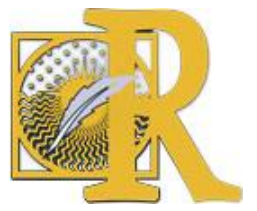


Institución que lo imparte.

Universidad Autónoma de Barcelona

Enlace: https://es.coursera.org/learn/pap

\section{Breve descripción.}

Este curso on-demand (ABIERTO, se puede cursar en cualquier momento), impartido en castellano por la Universidad Autónoma de Barcelona y el Centro de Crisis de Barcelona, está destinado a entrenar en la aplicación de primeros auxilios psicológicos (PAP) a personas afectadas por situaciones altamente estresantes, abarcando tanto emergencias cotidianas (incidentes críticos estadísticamente frecuentes que afectan de manera muy intensa: un accidente de tráfico, una hospitalización, una agresión o la muerte traumática o repentina de una persona, etc.) como emergencias comunitarias y/o masivas (sucesos infrecuentes, que afectan a muchas personas o a una comunidad entera y que sobrepasan con mucho lo que sucede habitualmente en ella: una catástrofe natural, un accidente ferroviario o aéreo o un atentado).

\section{Conclusiones.}

A la vista de todo lo presentado en este trabajo, consideramos que las MOOC posibilitan una nueva manera de concebir la enseñanza en el ámbito no presencial, abriendo un campo de posibilidades educativas que las tecnologías actuales ponen a disposición del sistema educativo formal y no formal.

Son muchas las iniciativas que podemos encontrar de actuación en este sentido, y como hemos podido ejemplificar, muy diversos los campos y temáticas a trabajar y las competencias a desarrollar en los discentes.

Dichas posibilidades supondrán un nuevo campo de investigación y de avance innovador que deberemos recorrer para garantizar el acceso al conjunto del alumnado y la formación adecuada de los docentes que se encarguen de su puesta en funcionamiento.

En este sentido, no debemos olvidar las posibilidades de una adecuada atención a la diversidad para lo cual dichas herramientas pueden servir de garante de respuesta a las necesidades particulares que en función de las diversas situaciones presente cada alumno o contexto educativo, pudiendo adaptarse a las mismas.

Así, tras lo aquí expuesto, concluimos que debemos avanzar hacia soluciones tecnológicas que den respuesta en la educación a la grave situación que se ha presentado ante el COVID19 y que demanda nuevas situaciones a afrontar.

En este sentido, los MOOC se presentan como una apuesta de calidad que posibilitará el establecimiento de nuevos mecanismos didácticos que nos hagan reaccionar ante las problemáticas de la pandemia, al tiempo que avanzaremos en la línea ya iniciada hace tiempo de dotar de nuevas potencialidades a la enseñanza en la sociedad del siglo XXI.

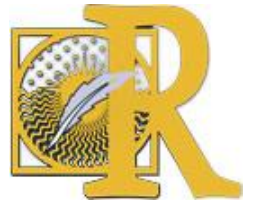




\section{Referencias bibliográficas}

- Bao, Y., Sun, Y., Meng, S., Shi, J., \& Lu, L. (2020). 2019-nCoV epidemic: address mental health care to empower society. The Lancet, 395(10224), 37-38.

- Brooks, S.K., Webster, R.K., Smith, L.E., Woodland, L., Wessely, S., Greenberg, N., \& Rubin, G.J. (2020). The Psychological Impact of Quarantine and How to Reduce It: Rapid Review of the Evidence. The Lancet, 395(10227), 912-20.

- Cabero-Almenara, J. \& Ruiz-Palmero, J. (2018). Las Tecnologías de la Información y Comunicación para la inclusión: reformulando la brecha digital. International Journal of Educational Research and Innovation (IJERI), 9, 16-30.

- Castaño-Muñoz, J., Duart, J. \& Teresa, S. (2015). Determinants of Internet use for interactive learning: an exploratory study. Journal of New Approaches in Educational Research, 4 (1), 25-34.

- Colorado, A., Marín-Díaz, V. \& Zavala, Z. (2016). Impacto del grado de apropiación tecnológica en los estudiantes de la Universidad Veracruzana. International Journal of Educational Research and Innovation (IJERI), 5, 124-137.

- Department for Business, Innovation and Skills (2013). The Maturing of the MOOC literature review of massive open online courses and other forms of online distance learning. London: Department for Business, Innovation and Skills.

- Downes, S. (2012). The Rise of MOOC. Recuperado de http://www.downes.ca/ post $/ 57911$.

- Downes, S. (2013). The Quality of Massive Open Online Courses. Recuperado de http://mooc.efquel.org/week-2-the-quality-of-massive-open-online-coursesby-stephendownes/.

- Estévez, J. \& García, A. (2015). Las redes sociales para la mejora de la capacidad de emprender y de autoempleo. International Journal of Educational Research and Innovation (IJERI), 4, 101-110.

- Gómez-Galán, J, Martín-Padilla, A. H., Bernal-Bravo, C., \& López-Meneses, E. (2017). Los MOOC y la Educación Superior Nuevas posibilidades para la innovación y la formación permanente. Barcelona: Octaedro

- Hill, P. (2012). Four Barriers that MOOCs must overcome to build a sustainable model. eLiterate. Recuperado de http://mfeldstein.com/four-barriers- hatmoocs-must-overcome-tobecome-sustainable-model/.

- Martín-Padilla, A.H., Bernal-Bravo, C., Ramírez-Fernández, M.B. \& López-Meneses, E. (2016). Propuesta para el diseño de un observatorio de calidad e innovación sobre MOOC. En López-Meneses, E., Cobos, D. \& Gómez Galán, J. (Eds.). La Educación Superior en el Siglo XXI: Una Reflexión desde y para el Profesorado, (pp. 35-46) Cupey: UMET Press/Innovagogía.

- Méndez García, C. (2015). Diseño e implementación de cursos abiertos masivos en línea (MOOC): expectativas y consideraciones prácticas. Revista De Educación a Distancia (RED), (39). Recuperado a partir de https://revistas.um.es/red/article/view/234251

- ONU. (2020). Nueve maneras en que la ONU apoya la lucha contra el coronavirus. Recuperado de: https://news.un.org/es/story/2020/04/1472832

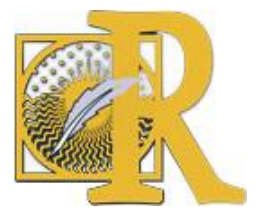


- Rheingold, H. (2013). MOOCs, Hype, and the Precarious State of Higher Ed: Futurist Bryan Alexander. Recuperado de: http://bit.ly/2qcFxgw

- Roig-Vila, R., Mondéjar, L. \& Lorenzo-Lledó, G. (2016). Redes sociales científicas. La Web social al servicio de la investigación. International Journal of Educational Research and Innovation (IJERI), 5, 171-183.

- Ruiz-Cruz, M.A. (2020). Los medios de comunicación y la cobertura de emergencias sociosanitarias. Fronteras de la Ciencia, 7, 54-61.

- SCOPEO (2013). SCOPEO INFORME NN2. MOOC: Estado de la situación actual, posibilidades, retos y futuro. Salamanca: Universidad de Salamanca-Centro Internacional de Tecnologías Avanzadas.

- Siemens, G. (2013). Massive Open Online Courses: Innovation in Education?. En R. McGreal, W. Kinuthia y S. Marshall (Eds.), Open Educational Resources: Innovation, Research and Practice (pp. 5-15). Vancouver: Commonwealth of Learning y Athabasca University.

- UNESCO. (2020a). Aprendiendo en casa: educación a distancia para todos. Recuperado de: https://es.unesco.org/news/aprendiendo-casa-educacion -distancia-todos

- UNESCO. (2020b). El coronavirus y la educación superior: impacto y recomendaciones. Recuperado de: https://www.iesalc.unesco.org/2020/ 04/02/el-coronavirus-covid-19-y-laeducacion-superior-impacto-y-reco mendaciones/

- UNICEF. (2020). LA EDUCACIÓN FRENTE AL COVID-19. Propuestas para impulsar el derecho a la educación durante la emergencia. Madrid: UNICEF España Dirección de Sensibilización y Políticas de Infancia Área de Educación en Derechos de Infancia y Ciudadanía Global. Recuperado de: https://www.unicef.es/sites/unicef.es/files/educa/unicef-educa-covid19-propuestasproteger-derecho-educacion-emergencia-0.pdf

- Vázquez-Cano, E. \& López-Meneses, E. (2015). La filosofía educativa de los MOOC y la educación universitaria. RIED. Revista Iberoamericana de Educación a Distancia, 18 (2), 25-37. Recuperado de: http://revistas.uned.es/index.php/ried/article/view/14261

- Vázquez-Cano, E., Gómez-Galán, J., Infante-Moro, A., \& López-Meneses, E. (2020). Incidence of a Non-Sustainability Use of Technology on Students' Reading Performance in Pisa. Sustainability, 12(2), 749. Recuperado de: https://doi.org/ 10.3390/su12020749

- Vázquez-Cano, E., López-Meneses, E., Méndez-Rey, J.M., Suárez-Guerrero, C., MartínPadilla, A.H., Román-Graván, P., Gómez-Galán, J. \& Revuelta-Domínguez, F.I. (2013): Guía didáctica sobre los MOOC. Sevilla: AFOE.

- Vázquez-Cano, E.; López Meneses, E., y Sarasola, J. L. (2013). La Expansión del Conocimiento en Abierto: Ios MOOCs. Barcelona: Octaedro.

${ }^{1}$ https://es.coursera.org/search?query=covid\&=y https://www.edx.org/search?q=covid

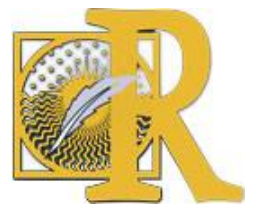

